\title{
Introduction of Near to Far Infrared Range Direct Band Gaps in Graphene: A First Principle Insight
}

\author{
Jeevesh Kumar, Ansh, and Mayank Shrivastava*
}

Cite This: ACS Omega 2021, 6, 5619-5626

Read Online

ABSTRACT: Lack of band gaps hinders application of graphene in the fields like logic, optoelectronics, and sensing despite its various extraordinary properties. In this work, we have done systematic investigations on direct band gap opening in graphene by hydrogenation and fluorination of carbon vacancies using the density functional theory computational approach. We have seen that although a carbon vacancy (void) opens an indirect band gap in graphene, it also creates unwanted mid gap (trap) states, which is attributed to unbound orbitals of the nearest unsaturated carbon

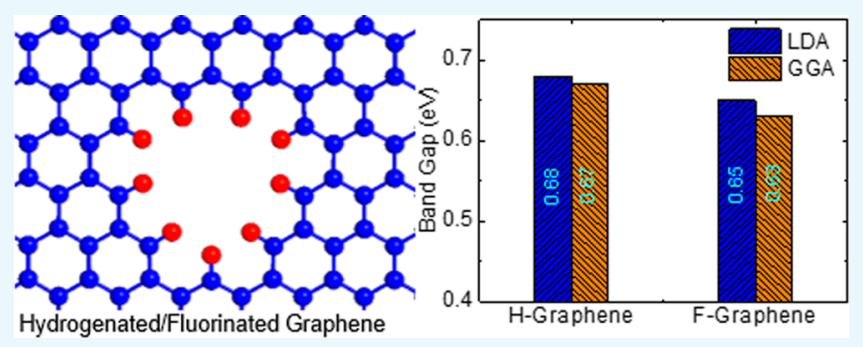
atoms at the vacant site. The unsaturated carbon atoms and corresponding trap states can degrade the stability of graphene and create band gaps particularly for large size vacancies. We have proposed that hydrogenation or fluorination of the unsaturated carbon atoms near the vacant site helps in disappearance of the trap states while contributing to promising direct band gaps in graphene. The opened band gap is tunable in the infrared regime and persists for different sizes and densities of hydrogenated or fluorinated patterns. In addition, we have also found that the proposed approach is thermodynamically favorable as well as stable. This keeps the planar nature of the graphene monolayer, despite creation of defects and subsequent functionalization, thereby making it useful for $2 \mathrm{D}$ material-based electronics, optoelectronics, and sensing applications.

\section{INTRODUCTION}

Carbon, in the form of graphene, ${ }^{1}$ has all the potential to replace conventional semiconductors due to its exceptional electrical, ${ }^{2}$ mechanical, ${ }^{3}$ optical, ${ }^{4}$ thermal, ${ }^{5}$ and magnetic ${ }^{6}$ properties. In spite of these remarkable properties, graphene is yet not acceptable for logic, optoelectronics, and sensing applications due to lack of band gaps. Various chemical and physical methods have been studied to create band gaps in graphene by breaking its lattice symmetry. Hydrogenation, proposed by Sofo et al., ${ }^{8}$ fluorination, proposed by Samarakoon et al. ${ }^{9}$ as well as Sahin et al., ${ }^{10}$ and oxygen plasma treatments, explored by Nourbakhsh et al., ${ }^{11}$ are some of the chemical methods in which external element changes hybridization of carbon from $\mathrm{sp}^{2}$ to $\mathrm{sp}^{3}$, which is attributed to the chemical interaction of external elements with graphene. This leads to creation of band gaps due to corresponding loss in symmetry. This however leads to loss of graphene's planar structure by converting graphene into graphane, fluorographene, and graphene oxide. Fan et al. have studied band gap opening in graphene by substitutional doping of boron and nitrogen; ${ }^{12}$ however, such a precise stoichiometry control would be experimentally tedious for corresponding substitutional doping of boron and nitrogen. Applications of external electric field ${ }^{13-15}$ and strain engineering ${ }^{16,17}$ are some of the physical methods demonstrated for band gap opening, which however are limited to band gaps that are in the $\mathrm{meV}$ range and hence could not be useful for majority of the applications.
Along with these methods, band gaps in graphene can also be opened by substrate interactions, ${ }^{18-21}$ metal interactions, ${ }^{22-26}$ doping, ${ }^{27,28}$ noncovalent interaction of molecules/chromophores, ${ }^{29,30}$ and quantum confinement; ${ }^{31}$ none of them however attracted engineering community due to limitations like loss of intrinsic graphene properties, nonplanar structure, thermodynamic instability, or complex processes.

Carbon vacancies (void engineering) in graphene are also a promising chemical method to create band gaps. ${ }^{32,33}$ Pedersen et al. $^{33}$ have discussed dependency of graphene band gaps on the density of the carbon vacancy (antidot). The authors however did not discuss how the band gap varies with antidot size. Behavior of trap states due to carbon vacancies and the stability of graphene with such antidots are also not discussed in detail in these earlier studies. It is worth highlighting that the unbound orbitals of the carbon atoms near antidots can easily react with ambient reactive gases like oxygen, which can change graphene's intrinsic properties and can also make it unstable. $^{34-36}$ Hence, a promising band gap engineering

Received: December 12, 2020

Accepted: February 9, 2021

Published: February 18, 2021 

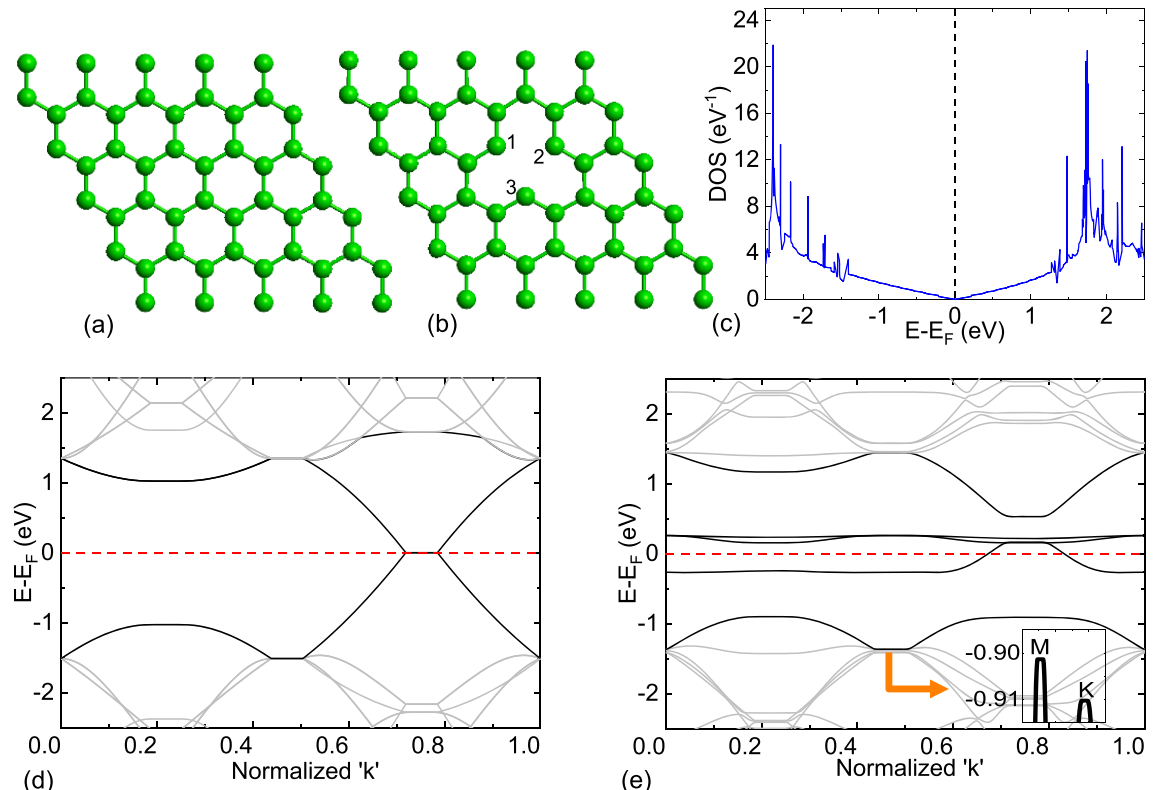

(e)

Figure 1. (a) $5 \times 5$ super cell of pristine graphene, (b) $5 \times 5$ super cell with the vacancy of one carbon atom (vac. graphene), (c) density of states (DOS) of pristine graphene, (d) band structure of the pristine graphene, and (e) band structure of the vac. Graphene. 1, 2, and 3 carbon atoms in (b) are the nearest neighbors of the vacant carbon atom. These are unsaturated $\mathrm{C}$ atoms due to the presence of unbound orbitals after the vacancy. Inset of (e) shows the zoomed in image of VBM, which shows that VBM at the M point is $\sim 0.01 \mathrm{eV}$ higher than VBM at the $\mathrm{K}$ point.

method in graphene is still in demand, which can give significant band gap tunability without noticeable distortion of its intrinsic properties as well as planar structure while offering a reliable and experimentally feasible approach. In this work, by using density functional theory (DFT)-based computations, we have done systematic investigations on vacancy-assisted band gap engineering of graphene while addressing reliability, stability, and mid-gap (trap) state-related issues using post defect hydrogenation and fluorination of vacancy sites. We have shown that an indirect band gap and mid-gap (trap) states are created in graphene due to carbon vacancies, which makes the material of lesser use particularly in the case of large vacancy size. Hydrogenation or fluorination of the vacancy site eliminates most of the trap states and converts an indirect band to a direct band, while almost retaining its intrinsic properties and the planar structure of graphene. The created direct band gap is in the near infrared regime, which can be tuned by varying the size and density of the vacancy pattern. In addition, it is also found to be environmentally stable as well as thermodynamically favorable.

\section{RESULTS AND DISCUSSION}

A $5 \times 5$ super cell of pristine graphene (Figure 1a) and the same super cell with one carbon vacancy (Figure $1 \mathrm{~b}$ ), also called vac. Graphene hereafter, were investigated. $\mathrm{C}-\mathrm{C}$ bond length in the pristine graphene is $\sim 1.42 \AA$, which is very close to a previously reported ${ }^{8,9}$ bond length value $(1.42 \AA)$. The density of states (DOS) and band structure of pristine graphene (Figure $1 \mathrm{c}, \mathrm{d}$ ) show zero band gap near the $\mathrm{K}$ point $(\mathrm{k}=$ 0.715 ), which, along with the $\mathrm{C}-\mathrm{C}$ bond length, validates our simulation setup used subsequently.

The carbon vacancy in graphene breaks its symmetry, which leaves unbound orbitals in the nearest carbon atoms $(1,2$, and 3 in Figure $1 b$ ) and makes them unsaturated. Perturbation in symmetry due to the vacancy breaks the Dirac cone and opens a band gap at the $\mathrm{K}$ point (Figure 1e). Valance band maxima (VBM) at the $\mathrm{M}$ point $(\mathrm{k}=0.172)$ is $\sim 0.01 \mathrm{eV}$ higher than
VBM at the $\mathrm{K}$ point (Figure 1e, inset), which indicates that the band gap created is indirect in nature with a value of $\sim 1.43 \mathrm{eV}$.

It is worth pointing out that the band structure with the introduced band gap has three intermediate/mid-gap energy states near the Fermi level. These mid-gap or trap states can alter the effective band gap, electrical, and optical properties of corresponding devices, like earlier shown for other 2D materials as studied by Ansh et al. ${ }^{37,38}$ and Jiang et al. ${ }^{39}$

Corresponding DOS analysis reveals that the unbound orbitals of the unsaturated carbon atoms near the vacant site have major contributions in the introduction of these mid-gap (trap) states (Figure 2).
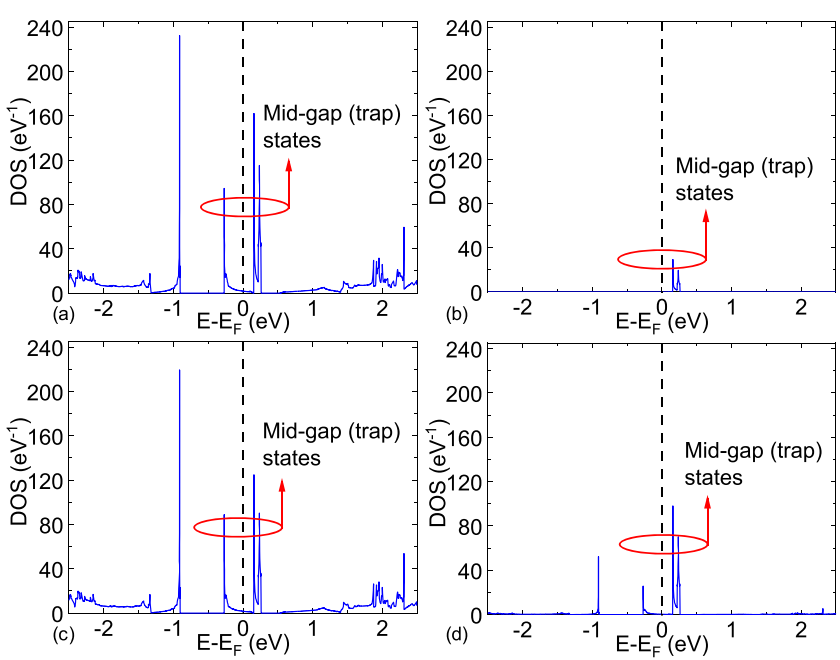

Figure 2. DOS of the $5 \times 5$ super cell with one carbon vacancy. (a) Total DOS, (b) DOS of s-orbitals of all the atoms in the super cell, (c) DOS of p-orbitals of all the atoms in the super cell, (d) DOS of porbitals of the three nearest atoms $(1,2$, and 3 in Figure $1 \mathrm{~b})$ at the vacancy site. Fermi level is at zero energy in all the plots. 
Comparison of DOS of the s-orbital and p-orbital (Figure $2 \mathrm{~b}, \mathrm{c}$ ) with the total DOS (Figure 2a) confirms that the midgap (trap) state's density near the Fermi level is majorly due to p-orbitals. Analysis of total DOS of the p-orbital (Figure 2c) vs DOS of the p-orbital due to the unsaturated carbon atoms (Figure 2d) confirms that the p-orbitals of the unsaturated carbon atoms have leading contribution in the mid-gap (trap) state's density near the Fermi energy.

Interaction between unsaturated carbon atoms of vac. Graphene and oxygen has been studied using PerdewBurke-Ernzerhof (PBE) form of the generalized gradient approximation (GGA) functional ${ }^{40}$ along with Grimme D2 van der Waals (vdW) correction. ${ }^{41}$ The same is compared with a similar study for pristine graphene (Figure 3 ).
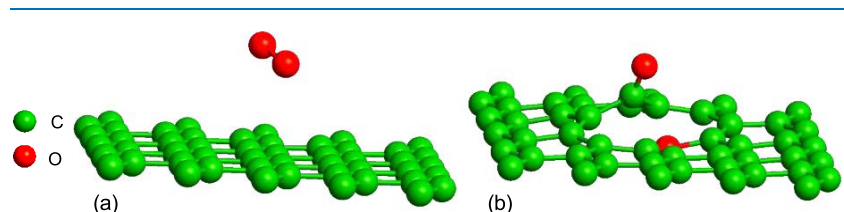

(b)

Figure 3. Energy optimized structures of (a) $5 \times 5$ super cell of pristine graphene with an oxygen molecule above it; (b) oxygen molecule near the graphene super cell with a carbon vacancy.

Carbon vacancies make the graphene reactive to oxygen at the vacant site, as also explored Kumar et al. in earlier wok. ${ }^{36}$ Oxygen is physically adsorbed over the pristine graphene surface (Figure 3a), whereas it dissociates over graphene near the vacant site due to availability of unbound orbitals. One of the dissociated oxygen atoms sits in the same plane as graphene, whereas the other atom sits above the graphene plane near the vacant site, which perturbs the planar structure of graphene (Figure $3 \mathrm{~b}$ ). Thus, vac. Graphene is thermodynamically less stable (more reactive) from its pristine counterpart in the presence of reactive gases like oxygen. These observations confirm that unsaturated carbon atoms of the vac. Graphene can initiate degradation of graphene near the vacant site and make it chemically unstable in the presence of the reactive agents like oxygen.

Hence, we can say that unwanted mid-gap (trap) states and oxidative sites due to the carbon vacancy makes the vacancy engineering unreliable for creating band gaps in graphene.

Hydrogenation and Fluorination. Passivation engineering of unbound orbitals while keeping the introduced band gap unaltered is the key to overcome the mid-gap (trap) states and oxidation of graphene. Monovalent nonmetal like hydrogen or halogen can be suitable agents, which can bond with unsaturated carbon atoms near the vacant site covalently and make them relatively inert. We propose to use hydrogen and/ or fluorine for passivation, which is due to their smaller sizes, thereby enabling them to stay in the same plane as graphene while leaving least strain after bonding.

Energy-optimized structures of the $5 \times 5 \times 1$ super cell of hydrogenated graphene (H-graphene) and fluorinated graphene (F-graphene), as shown in Figure 4a,b, respectively, show that the hydrogen and fluorine atoms deform nearby rings of the vacant site. $\mathrm{C}-\mathrm{H}$ bond length and $\mathrm{C}-\mathrm{F}$ bond length in the corresponding modules are $\sim 1.03$ and $\sim 1.27 \AA$, whereas the corresponding formation energies are -14.05 and - $3.15 \mathrm{eV}$, respectively. Negative formation energies, calculated using eq 1 below $(n=3)$, confirm that although hydrogenation and fluorination strained and disordered the
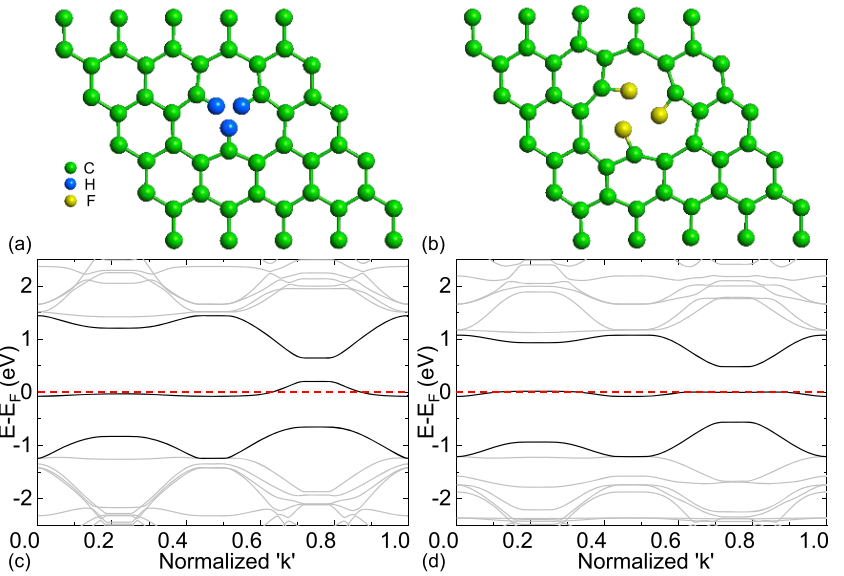

Figure 4. (a) Hydrogenated $5 \times 5$ super cell of graphene at one carbon vacancy, (b) fluorinated $5 \times 5$ super cell of graphene at one carbon vacancy, (c) band structure of the hydrogenated graphene, and (d) band structure of the fluorinated graphene.

nearest hexagonal rings in graphene (Figure 4a,b), the proposed passivation processes are thermodynamically stable and favorable for vac. Graphene with hydrogen or fluorine plasma. Disorder in the hexagonal rings due to fluorination is significant due to large size of the fluorine atom, which can be mitigated by increasing vacancy size in the graphene film.

$$
\begin{aligned}
& \Delta E_{\text {formation }}=E_{\text {final system }}-\left(n^{*} E_{\text {hydrogen or fluorine atom }}\right. \\
& \left.\quad+E_{\text {vac. Graphene }}\right) \\
& \text { ' } n \text { ' is the number of hydrogen or fluorine atoms in the system }
\end{aligned}
$$

Band gaps of hydrogenated and fluorinated graphene were found to be $\sim 1.3$ and $\sim 1.04 \mathrm{eV}$, respectively, which are direct in nature at the $\mathrm{K}$ point (Figure $4 \mathrm{c}, \mathrm{d}$ ). Two of the three midgap (trap) states of vac. Graphene disappeared after introducing $\mathrm{H}$ - or F-based passivation. Although one midgap (trap) state, mainly due to contributions of p-orbitals of all the carbon atoms, still exists near the Fermi level as shown in the band structure (Figure 4c,d) and in the corresponding DOS (Figure S2) plots, overall mid-gap (trap) state effect in the vac. Graphene is significantly reduced due to hydrogenation and fluorination. Hydrogenated and fluorinated graphene are relatively inert as well, in the presence of ambient oxygen due to unavailability of vacant orbitals near the defected sites, which has been discussed later.

Band structures of $5 \times 5$ super cells of hydrogenated graphene and fluorinated graphene have also been calculated using the GGA functional (Figure S3) and compared with corresponding local density approximation (LDA) results. However, fluorinated graphene shows deformation in the planar structure to minimize the ring strain (Figure S3), which however does not persist once vacancy size is increased as shown later in Figure 10. Comparable band structure and band gap results of the LDA functional with the GGA functional confirm the validity of the LDA approach, which has been used in further explorations to save computational time especially for larger super cells.

Band Gap Vs Pattern Density. We have explored a $5 \times 5$ super cell above, which has one carbon vacancy out of 50 carbon atoms. To understand the effect of different vacancy (pattern) densities over the band gap, super cell size has been 
increased to $7 \times 7$, which has one carbon vacancy out of 98 carbon atoms in the super cell (Figure S4a).

Hydrogenated and fluorinated graphene structures (optimized for minimum energy), as shown in Figure S4b,c, and corresponding band structures (Figure $5 \mathrm{~b}, \mathrm{c}$ ) follow a similar
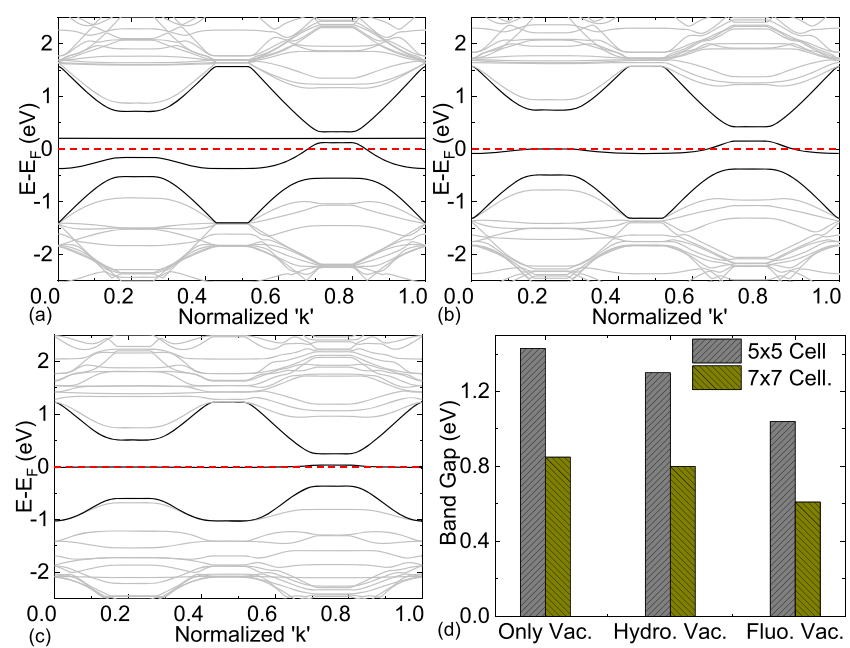

Figure 5. (a) Band structure of a $7 \times 7$ super cell of graphene with one carbon vacancy, (b) band structure of the hydrogenated graphene, (c) band structure of the fluorinated graphene, (d) band gap comparison of hydrogenated, fluorinated, and only vac. Graphene with different cell sizes (pattern density).

trend as depicted by the $5 \times 5$ super cell. The band gap of graphene with only one vacancy is indirect (Figure 5a), while the hydrogenated and fluorinated structures have a direct band gap. A lesser band gap in the $7 \times 7$ super cell as compared to the $5 \times 5$ super cell of all the modules (Figure $5 \mathrm{~d}$ ) confirms that the band gap in the system decreases with a decrease in hydrogenated or fluorinated pattern density in graphene.

Band Gap Vs Pattern Size. Knocking off one carbon atom from graphene to create vacancies is a very tedious process experimentally. To make the study experimental compatible, the band gap behavior of graphene with large vacancy size has been explored and analyzed. An $11 \times 11$ graphene super cell has been selected for this study so that large size vacancies can be created for further exploration. The super cell with a 4-C vacancy and $13-\mathrm{C}$ vacancy has been optimized for minimum energy (Figure 6a,b), and corresponding band structures have been computed as shown in Figure $6 \mathrm{c}, \mathrm{d}$, respectively.

Band structures of both these modules (Figure 6c,d) show that multiple mid-gap (trap) states are available in the mid gap region of the forbidden gap, which affects the usability of the introduced band gap in graphene. The mid-gap (trap) states are due to multiple unbound orbitals of the carbon atoms near the vacant site as discussed in the previous section. Hence, the large size vacancy created in the experimental process would also not produce a clear band gap in the graphene.

Hydrogenation or fluorination of the carbon atoms near the vacant site gives a direct band gap and abolishes most of the mid-gap (trap) states due to $\mathrm{C}-\mathrm{H}$ or $\mathrm{C}-\mathrm{F}$ bonding, which saturates all the unbound orbitals (Figure 7). Remaining midgap (trap) states, which are almost on the Fermi level, do not degrade the corresponding device's electronic properties significantly due to their deep levels and flat curvatures. Preferred (Figure S5) one-to-one bonding of hydrogen and fluorine atoms with the unsaturated carbons is in the plane of
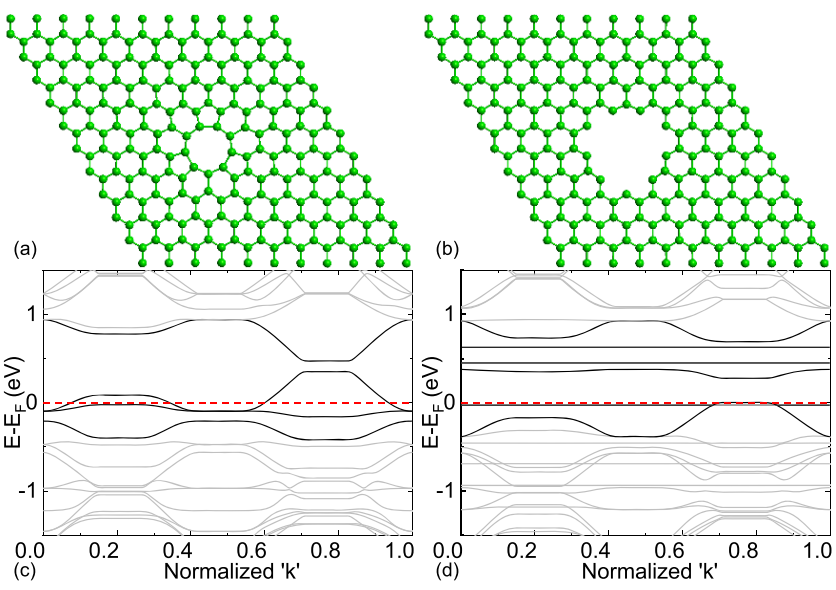

Figure 6. (a) $11 \times 11$ Super cell of graphene with a 4-C vacancy, (b) $11 \times 11$ super cell of graphene with a 13-C vacancy, (c) band structure of the 4C-vacancy module, and (d) band structure of the $13 \mathrm{C}$-vacancy module.

graphene, which validates the presence of $\mathrm{sp}^{2}$ hybridization necessary to preserve the planar structure after the bonding process. The super cells of different vacancy sizes have different band gap values (Figure 8a). Variation in vacancy size can give a knob to play with band gap values to make different devices for different applications. Large vacancy size provides enough space for bonded hydrogen or fluorine atoms to stay relaxed in the graphene plane without introducing distortion or strain into the neighboring hexagons (Figure $7 \mathrm{e}, \mathrm{f}$ ), which gives a relatively strain-free environment unlike the corresponding small size vacancy case (Figure $4 \mathrm{a}, \mathrm{b}$ ).

Formation energies of hydrogenation and fluorination (Figure $8 \mathrm{~b}$ ) processes are calculated using eq 1 . The negative value of formation energy signifies that the processes are thermodynamically favorable to replicate experimentally using hydrogen or fluorine ions. Formation energy per atom increases with an increase in the vacancy due to reduction in the strain energy of the system having large vacancy size. When vacancy size is increased, fluorination gains significant formation energy compared to hydrogenation because multiple fluorine atoms do not sit like hydrogen atoms in the small vacancy due to their large sizes. When vacancy size is increased, fluorine atoms can have enough stress-free space, which increases corresponding formation energy significantly due to reduction in strain energy.

Unsaturated carbon due to the vacancy in graphene shows one-to-one bonding tendency to hydrogen/fluorine in the presence of corresponding molecules (Figure S5). One-to-one bonding does not leave any unsaturated orbital near the vacant site. Lack of unsaturated orbitals should make the system relatively stable in the presence of external chemically reactive agents like oxygen. To explore this, interactions of the $11 \times 11$ super cells (13 $\mathrm{C}$ vacancy) of $\mathrm{H}$-Graphene and F-Graphene with oxygen have been studied using PBE with Grimme D2 van der Waals (vdW) correction.

Oxygen is physically adsorbed over the surfaces near hydrogenated (Figure 9a) or fluorinated (9b) sites. It does not dissociate, like we have seen earlier (Figure 3), over vac. Graphene. The planar structure of graphene is also preserved in the presence of oxygen near the hydrogenated site, while it is perturbed slightly near the fluorinated site due to loan pairloan pair interactions of oxygen and fluorine (Figure S6). 

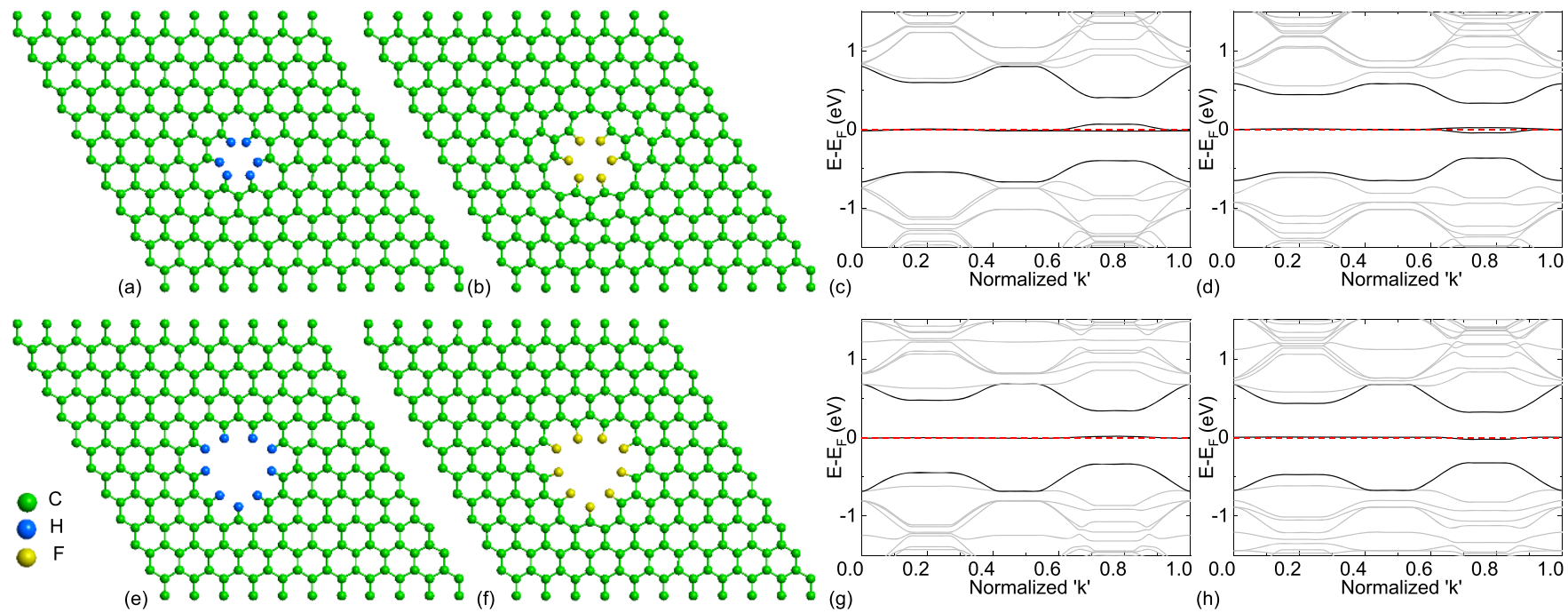

Figure 7. (a) Hydrogenated $11 \times 11$ super cell of graphene with a 4-C vacancy, (b) fluorinated $11 \times 11$ super cell of graphene with a 4-C vacancy, (c) band structure of the module in (a), and (d) band structure of the module in (b). (e) Hydrogenated 11x11 super cell of graphene with a 13-C vacancy, $(\mathrm{f})$ fluorinated $11 \times 11$ super cell of graphene with a 13-C vacancy, $(\mathrm{g})$ band structure of the module in $(\mathrm{e})$, and $(\mathrm{h})$ band structure of the module in (f).
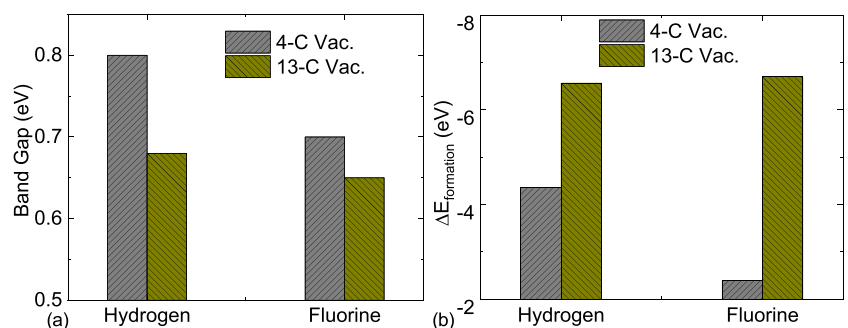

Figure 8. (a) Band gap and (b) formation energy of the $11 \times 11$ super cell of hydrogenated and fluorinated graphene with different vacancy sizes.
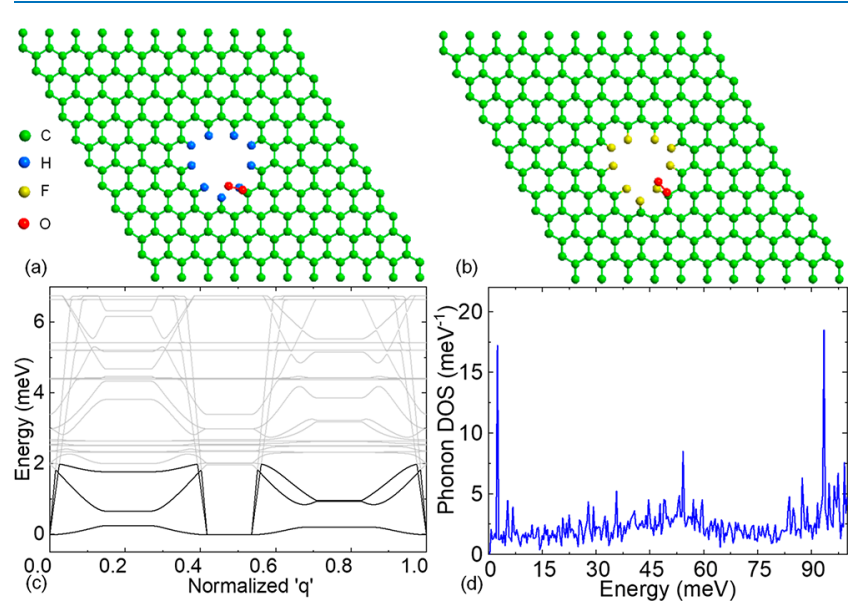

Figure 9. Energy-optimized structures of (a) hydrogenated graphene with an oxygen molecule and (b) fluorinated graphene with an oxygen molecule. (c) Phonon band structure and (d) phonon density of states of hydrogenated graphene $(1 \mathrm{C}$ Vac. $11 \times 11$ super cell)

Oxygen is physically adsorbed over the surfaces near hydrogenated (Figure 9a) or fluorinated (9b) sites. It does not dissociate, like we have seen earlier (Figure 3), over vac. Graphene. The planar structure of graphene is also preserved in the presence of oxygen near the hydrogenated site, while it is perturbed slightly near the fluorinated site due to loan pairloan pair interactions of oxygen and fluorine (Figure S6).

Nonnegative energy values of phonon bands (Figure 9c,d) calculated using the classical ForceField method ${ }^{42}$ with the Tersoff potential profile ${ }^{43}$ confirm that graphene with a hydrogenated vacancy is thermodynamically stable. Fluorinated graphene can also be predicted to be thermodynamically stable due to the similar structure of hydrogenated graphene and existence of the $\mathrm{F}-\mathrm{C}-\mathrm{C}-$ bond in the nature. The corresponding phonon band structure has not been discussed here due to computational limitations.

To validate the LDA approach, band structures of $11 \times 11$ super cells of hydrogenated graphene and fluorinated graphene have been calculated (Figure 10) using the GGA functional and compared with corresponding LDA results. Comparable planar structures and band gaps of hydrogenated graphene and fluorinated graphene using LDA and GGA methods confirm that hydrogenation or fluorination of the carbon vacancy can be a promising process to create a near infrared range direct band gap in graphene.

We also investigated the chlorine atom $(\mathrm{Cl})$ from the halogen family for passivation. Despite similar chemistry, chlorine was found not to be useful for introduction of band gaps in graphene (Figure S7). Although it created energy gap at the Dirac point, the Fermi level was found to lie inside the conduction band, which makes the vac. Graphene-chlorine system metallic. Bromine $(\mathrm{Br})$ and iodine(I) are not investigated for passivation due to their very large sizes as compared to carbon.

Finally, it is also worth highlighting that vacancy creation in graphene was recently demonstrated experimentally by $\mathrm{A}$. Meersha et al. ${ }^{44}$ by using the following approaches: (i) Ar-ion bombardment or oxygen-ion bombardment inside an ultrahigh vacuum chamber, which is otherwise used for surface cleaning post lithography, (ii) Ar plasma or oxygen plasma treatment using a reactive ion etching system, and (iii) local burning/damage of the graphene lattice using an electron gun inside an e-beam lithography system. Ar bombardment or Arion treatment knocks of the carbon atom from the graphene lattice due to its large size. This is physical removal of the 
(a)

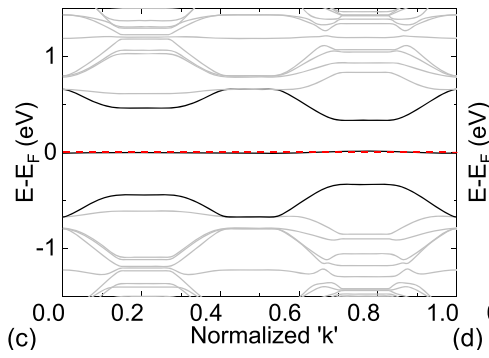

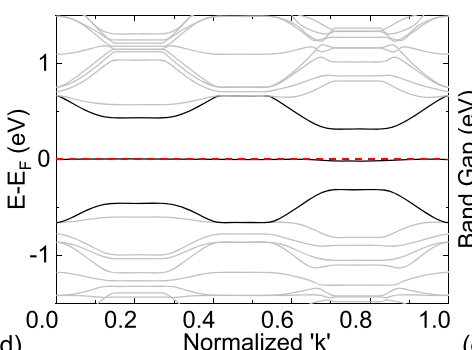

(d)
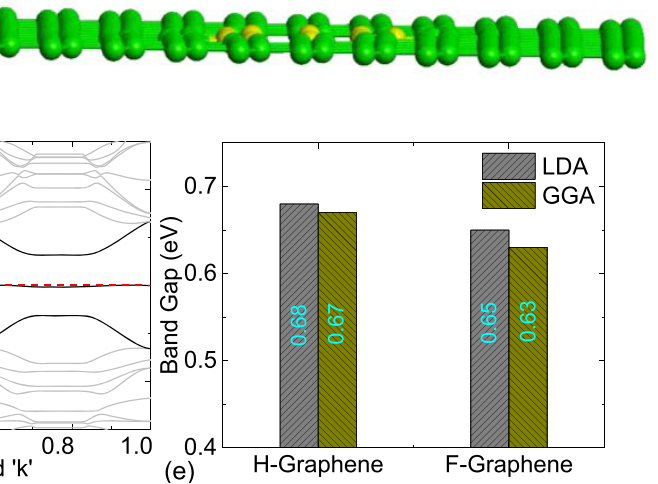

Figure 10. Tilted side view of (a) hydrogenated and (b) fluorinated 13 carbon vacancy of a 11x11 graphene super cell after optimization using PBE form of the GGA functional. Band structures of $11 \times 11$ supercells of (c) H-graphene and (d) F-graphene, computed using PBE form of the GGA functional. (e) Band gap comparison with the LDA functional.

carbon atom. Oxygen bombardment or oxygen-ion treatment leads to chemical removal of the carbon atom. These two however produce defects, which are statistically distributed and indeterministic. On the other hand, an electron gun-based approach can be used for patterning vacancy/defect regions in graphene in a deterministic fashion. In addition, use of a highenergy $(>20 \mathrm{KeV})$ electron gun can allow defect size of less than $1 \mathrm{~nm}$. Similar approaches can be extended for hydrogen/ fluorine plasma-based processing to create the hydrogenated/ fluorinated pattern in graphene for desired band gaps.

\section{CONCLUSIONS}

We have shown that a band gap created due to the carbon vacancy in graphene is not practically useful due to the introduction of mid-gap (trap) states and affinity of the vacant site with oxygen present in an ambient atmosphere. Hydrogenation or fluorination of the carbon vacancy has been subsequently shown to be a promising method, which eliminates most of the trap states while keeping the introduced band gap unchanged. The proposed process also overcomes the reactivity issue of graphene with ambient oxygen. In addition, while the vacancy-assisted band gap was found to be indirect, hydrogenation or fluorination of the vacancy site converted it to a direct band gap, tunable into the infrared regime. The findings persist for different vacancy sizes as well as densities of the vacancy pattern. Furthermore, the bonded hydrogen or fluorine atoms at vacancy sites were found to be in the same plane as graphene, which helps to preserve its planar structure. We have also shown that the proposed processes have negative formation energies, which indicates that it is thermodynamically favorable for experimental replications using hydrogen or fluorine plasma. These findings therefore show usefulness of the proposed approach for electronics, optoelectronics, and sensing applications.

\section{COMPUTATIONAL METHODS}

All investigations were done using the $\mathrm{DFT}^{45}$-based quantum computations supported by the QuantumATK package. ${ }^{46-48}$ For DFT calculation, the super cells were optimized with 0.01 $\mathrm{eV} / \AA$ force and $0.001 \mathrm{eV} / \AA^{3}$ energy cutoffs. $\mathrm{LDA}^{49}$ was used in most of the computations to utilize optimum computation time for larger cells. However, the computed results were also validated using PBE form of the GGA functional. ${ }^{40,41} 10 \times 10 \times 1$ $\mathrm{k}$ point sampling (Figure 1) for energy optimization and band structure calculation and $15 \times 15 \times 1 \mathrm{k}$ point sampling for DOS calculations were used along with a density mess cutoff of 75 Hartree. Periodic boundary conditions were applied in all the calculations to emulate a large area graphene sheet. $\sim 15 \AA$ vacuum regions were added to both sides of the graphene plane to eliminate wave function interaction of alternate planes in periodic boundary conditions. Band structures are plotted with normalized ' $\kappa$ ', which is along $\mathrm{G} \rightarrow \mathrm{M} \rightarrow \mathrm{L} \rightarrow \mathrm{A} \rightarrow \mathrm{G} \rightarrow$ $\mathrm{K} \rightarrow \mathrm{H} \rightarrow \mathrm{A}$. The positions of $\mathrm{G}, \mathrm{M}, \mathrm{L}, \mathrm{A}, \mathrm{G}, \mathrm{K}, \mathrm{H}$, and $\mathrm{A}$ points on the $\mathrm{k}$ axis are $0,0.172,0.258,0.430,0.516,0.715$, 0.801 , and 1.0 , respectively. Fermi energy has been set to zero in all the band structure and DOS plots. In the band structure images, bands near the Fermi level are in dark color. Other bands of the band structures are in gray shade so that the bands near the Fermi level can be distinguished easily.

\section{ASSOCIATED CONTENT}

\section{Supporting Information}

The Supporting Information is available free of charge at https://pubs.acs.org/doi/10.1021/acsomega.0c06058.

Plot of the k-point convergent test; DOS of hydrogenated and fluorinated graphene; LDA vs GGA band structure comparison of hydrogenated and fluorinated graphene; optimized structures of $7 \times 7$ graphene super cells; $\mathrm{H}_{2}$ and $\mathrm{F}_{2}$ near the vacancy site of graphene; optimized structures of $\mathrm{O}_{2}$ near hydrogenated and fluorinated graphene; chlorinated carbon vacancies in graphene (PDF).

\section{AUTHOR INFORMATION}

\section{Corresponding Author}

Mayank Shrivastava - Department of Electronic Systems Engineering, Indian Institute of Science, Bangalore 560012, India; Phone: 09591140309; Email: mayank@iisc.ac.in

\section{Authors}

Jeevesh Kumar - Department of Electronic Systems

Engineering, Indian Institute of Science, Bangalore 560012, India; 1 orcid.org/0000-0001-6178-8434

Ansh - Department of Electronic Systems Engineering, Indian Institute of Science, Bangalore 560012, India

Complete contact information is available at: https://pubs.acs.org/10.1021/acsomega.0c06058 


\section{Notes}

The authors declare no competing financial interest.

\section{ACKNOWLEDGMENTS}

The authors would like to acknowledge the NNetRA program of MeitY, DST, and CSIR, as well as DRDO and MHRD Govt. of India, for supporting this work.

\section{REFERENCES}

(1) Novoselov, K. S.; Geim, A. K.; Morozov, S. V.; Jiang, D.; Zhang, Y.; Dubonos, S. V.; Grigorieva, I. V.; Firsov, A. A. Electric Field Effect in Atomically Thin Carbon Films. Science 2004, 306, 666-669.

(2) Morozov, S. V.; Novoselov, K. S.; Katsnelson, M. I.; Schedin, F.; Elias, D. C.; Jaszczak, J. A.; Geim, A. K. Giant Intrinsic Carrier Mobilities in Graphene and Its Bilayer. Phys. Rev. Lett. 2008, 100, 1114.

(3) Lee, C.; Wei, X.; Kysar, J. W.; Hone, J. Measurments of the Elastic Properties and Intrinsic Strength of Monolayer Graphene. Science 2008, 321, 385-388.

(4) Falkovsky, L. A. Optical Properties of Graphene. J. Phys. Conf. Ser. 2008, 129, No. 012004.

(5) Balandin, A. A.; Ghosh, S.; Bao, W.; Calizo, I.; Teweldebrhan, D.; Miao, F.; Lau, C. N. Superior Thermal Conductivity of SingleLayer Graphene. Nano Lett. 2008, 8, 902-907.

(6) Ramakrishna Matte, H. S. S.; Subrahmanyam, K. S.; Rao, C. N. R. Novel Magnetic Properties of Graphene: Presence of Both Ferromagnetic and Antiferromagnetic Features and Other Aspects. J. Phys. Chem. C 2009, 113, 9982-9985.

(7) Xu, X.; Liu, C.; Sun, Z.; Cao, T.; Zhang, Z.; Wang, E.; Liu, Z.; Liu, K. Interfacial Engineering in Graphene Bandgap. Chem. Soc. Rev. 2018, 47, 3059-3099.

(8) Sofo, J. O.; Chaudhari, A. S.; Barber, G. D. Graphane: A TwoDimensional Hydrocarbon. Phys. Rev. B 2007, 75, 1-4.

(9) Samarakoon, D. K.; Chen, Z.; Nicolas, C.; Wang, X. Q. Structural and Electronic Properties of Fluorographene. Small 2011, 7, 965-969.

(10) Åžahin, H.; Topsakal, M.; Ciraci, S. Structures of Fluorinated Graphene and Their Signatures. Phys. Rev. B-Condens. Matter Mater. Phys. 2011, 83, 1-6.

(11) Nourbakhsh, A.; Cantoro, M.; Vosch, T.; Pourtois, G.; Clemente, F.; Van Der Veen, M. H.; Hofkens, J.; Heyns, M. M.; De Gendt, S.; Sels, B. F. Bandgap Opening in Oxygen Plasma-Treated Graphene. Nanotechnology 2010, 21, No. 435203.

(12) Fan, X.; Shen, Z.; Liu, A. Q.; Kuo, J. L. Band Gap Opening of Graphene by Doping Small Boron Nitride Domains. Nanoscale 2012, 4, 2157-2165.

(13) Zhang, Y.; Tang, T. T.; Girit, C.; Hao, Z.; Martin, M. C.; Zettl, A.; Crommie, M. F.; Shen, Y. R.; Wang, F. Direct Observation of a Widely Tunable Bandgap in Bilayer Graphene. Nature 2009, 459, $820-823$.

(14) Oostinga, J. B.; Heersche, H. B.; Liu, X.; Morpurgo, A. F.; Vandersypen, L. M. K. Gate-Induced Insulating State in Bilayer Graphene Devices. Nat. Mater. 2008, 7, 151-157.

(15) Castro, E. V.; Novoselov, K. S.; Morozov, S. V.; Peres, N. M. R.; Dos Santos, J. M. B. L.; Nilsson, J.; Guinea, F.; Geim, A. K.; Neto, A. H. C. Biased Bilayer Graphene: Semiconductor with a Gap Tunable by the Electric Field Effect. Phys. Rev. Lett. 2007, 99, 8-11.

(16) Gui, G.; Li, J.; Zhong, J. Band Structure Engineering of Graphene by Strain: First-Principles Calculations. Phys. Rev. BCondens. Matter Mater. Phys. 2008, 78, 1-6.

(17) Ni, Z. H.; Yu, T.; Lu, Y. H.; Wang, Y. Y.; Feng, Y. P.; Shen, Z. $\mathrm{X}$. Uniaxial Strain on Graphene: Raman Spectroscopy Study and Band-Gap Opening. ACS Nano 2008, 2, 2301-2305.

(18) Fan, X. F.; Zheng, W. T.; Chihaia, V.; Shen, Z. X.; Kuo, J. L. Interaction between Graphene and the Surface of SiO2. J. Phys. Condens. Matter 2012, 24, No. 305004.

(19) Mattausch, A.; Pankratov, O. Ab Initio Study of Graphene on SiC. Phys. Rev. Lett. 2007, 99, 1-4.
(20) Varchon, F.; Feng, R.; Hass, J.; Li, X.; Nguyen, B. N.; Naud, C.; Mallet, P.; Veuillen, J. Y.; Berger, C.; Conrad, E. H.; Magaud, L. Electronic Structure of Epitaxial Graphene Layers on SiC: Effect of the Substrate. Phys. Rev. Lett. 2007, 99, 3-6.

(21) Nevius, M. S.; Conrad, M.; Wang, F.; Celis, A.; Nair, M. N.; Taleb-Ibrahimi, A.; Tejeda, A.; Conrad, E. H. Semiconducting Graphene from Highly Ordered Substrate Interactions. Phys. Rev. Lett. 2015, 115, 1-5.

(22) Giovannetti, G.; Khomyakov, P. A.; Brocks, G.; Karpan, V. M.; Van Den Brink, J.; Kelly, P. J. Doping Graphene with Metal Contacts. Phys. Rev. Lett. 2008, 101, 4-7.

(23) Gong, C.; Lee, G.; Shan, B.; Vogel, E. M.; Wallace, R. M.; Cho, K. First-Principles Study of Metal-Graphene Interfaces. J. Appl. Phys. 2010, 108, No. 123711.

(24) Khomyakov, P. A.; Giovannetti, G.; Rusu, P. C.; Brocks, G.; Van Den Brink, J.; Kelly, P. J. First-Principles Study of the Interaction and Charge Transfer between Graphene and Metals. Phys. Rev. BCondens. Matter Mater. Phys. 2009, 79, 1-12.

(25) Voloshina, E.; Dedkov, Y. Graphene on Metallic Surfaces: Problems and Perspectives. Phys. Chem. Chem. Phys. 2012, 14, 13502-13514.

(26) Vita, H.; Böttcher, S.; Horn, K.; Voloshina, E. N.; Ovcharenko, R. E.; Kampen, T.; Thissen, A.; Dedkov, Y. S. Understanding the Origin of Band Gap Formation in Graphene on Metals: Graphene on $\mathrm{Cu} / \mathrm{Ir}(111)$. Sci. Rep. 2015, 4, 1-8.

(27) Denis, P. A. Band Gap Opening of Monolayer and Bilayer Graphene Doped with Aluminium, Silicon, Phosphorus, and Sulfur. Chem. Phys. Lett. 2010, 492, 251-257.

(28) Mohammed, M. H. Designing and Engineering Electronic Band Gap of Graphene Nanosheet by P Dopants. Solid State Commun. 2018, 258, 11-16.

(29) Joo, Y.; Kim, M.; Kanimozhi, C.; Huang, P.; Wong, B. M.; Singha Roy, S.; Arnold, M. S.; Gopalan, P. Effect of Dipolar Molecule Structure on the Mechanism of Graphene-Enhanced Raman Scattering. J. Phys. Chem. C 2016, 120, 13815-13824.

(30) Huang, C.; Kim, M.; Wong, B. M.; Safron, N. S.; Arnold, M. S.; Gopalan, P. Raman Enhancement of a Dipolar Molecule on Graphene. J. Phys. Chem. C 2014, 118, 2077-2084.

(31) Son, Y. W.; Cohen, M. L.; Louie, S. G. Energy Gaps in Graphene Nanoribbons. Phys. Rev. Lett. 2006, 97, 1-4.

(32) Dvorak, M.; Oswald, W.; Wu, Z. Bandgap Opening by Patterning Graphene. Sci. Rep. 2013, 3, 1-7.

(33) Pedersen, T. G.; Flindt, C.; Pedersen, J.; Mortensen, N. A.; Jauho, A. P.; Pedersen, K. Graphene Antidot Lattices: Designed Defects and Spin Qubits. Phys. Rev. Lett. 2008, 100, 1-4.

(34) Pašti, I. A.; Jovanović, A.; Dobrota, A. S.; Mentus, S. V.; Johansson, B.; Skorodumova, N. V. Atomic Adsorption on Graphene with a Single Vacancy: Systematic DFT Study through the Periodic Table of Elements. Phys. Chem. Chem. Phys. 2018, 20, 858-865.

(35) Jovanović, A. Z.; Mentus, S. V.; Skorodumova, N. V.; Pašti, I. A. Reactivity Screening of Single Atoms on Modified Graphene Surface: From Formation and Scaling Relations to Catalytic Activity. Adv. Mater. Interfaces 2020, 2001814, 2001814-2001813.

(36) Kumar, J.; Ansh; Shrivastava, M. Stone-Wales Defect and Vacancy-Assisted Enhanced Atomic Orbital Interactions between Graphene and Ambient Gases: A First-Principles Insight. ACS Omega 2020, 5, 31281-31288.

(37) Ansh; Kumar, J.; Sheoran, G.; Variar, H. B.; Mishra, R.; Kuruva, H.; Meersha, A.; Mishra, A.; Raghavan, S.; Shrivastava, M. ChalcogenAssisted Enhanced Atomic Orbital Interaction at TMD-Metal Interface and Sulfur Passivation for Overall Performance Boost of 2-D TMD FETs. IEEE Trans. Electron Devices 2020, 67, 717-724.

(38) Ansh; Kumar, J.; Sheoran, G.; Mishra, R.; Raghavan, S.; Shrivastava, M. Selective Electron or Hole Conduction in Tungsten Diselenide (WSe2) Field-Effect Transistors by Sulfur-Assisted MetalInduced Gap State Engineering. IEEE Trans. Electron Devices 2020, 67, 383-388.

(39) Jiang, J.; Ling, C.; Xu, T.; Wang, W.; Niu, X.; Zafar, A.; Yan, Z.; Wang, X.; You, Y.; Sun, L.; Lu, J.; Wang, J.; Ni, Z. Defect Engineering 
for Modulating the Trap States in 2D Photoconductors. Adv. Mater.

2018, 30, 1-6.

(40) Perdew, J. P.; Burke, K.; Ernzerhof, M. Generalized Gradient Approximation Made Simple. Phys. Rev. Lett. 1996, 77, 3865-3868.

(41) Grimme, S. Semiempirical GGA-Type Density Functional Constructed with a Long-Range Dispersion Correction. J. Comput. Chem. 2006, 27, 1787-1799.

(42) Schneider, J.; Hamaekers, J.; Chill, S. T.; Smidstrup, S.; Bulin, J.; Thesen, R.; Blom, A.; Stokbro, K. ATK-ForceField: A New Generation Molecular Dynamics Software Package. arXiv 2017, 25, No. 085007.

(43) Lindsay, L.; Broido, D. A. Optimized Tersoff and Brenner Empirical Potential Parameters for Lattice Dynamics and Phonon Thermal Transport in Carbon Nanotubes and Graphene. Phys. Rev. BCondens. Matter Mater. Phys. 2010, 81, 1-6.

(44) Meersha, A.; Variar, H. B.; Bhardwaj, K.; Mishra, A.; Raghavan, S.; Bhat, N.; Shrivastava, M. Record Low Metal - (CVD) Graphene Contact Resistance Using Atomic Orbital Overlap Engineering. Tech. Dig. Int. Electron Devices Meet. IEDM 2017, No. Cvd, 5.3.1-5.3.4. DOI: $10.1109 /$ IEDM.2016.7838352

(45) Kohn, W.; Sham, L. J. Self-Consistent Equations Including Exchange and Correlation Effects. Phys. Rev. 1965, 140, No. A1133.

(46) QuantumATK O-2018.06.

(47) Soler, J. M.; Artacho, E.; Gale, J. D.; García, A.; Junquera, J.; Ordejón, P.; Sánchez-Portal, D. The SIESTA Method for Ab Initio Order-N Materials Simulation. J. Phys. Condens. Matter 2002, 14, 2745-2779.

(48) Smidstrup, S.; Stradi, D.; Wellendorff, J.; Khomyakov, P. A.; Vej-Hansen, U. G.; Lee, M. E.; Ghosh, T.; Jónsson, E.; Jónsson, H.; Stokbro, K. First-Principles Green's-Function Method for Surface Calculations: A Pseudopotential Localized Basis Set Approach. Phys. Rev. B 2017, 96, 1-17.

(49) Perdew, J. P.; Zunger, A. Self-Interaction Correction to Density-Functional Approximations for Many-Electron Systems. Phys. Rev. B 1981, 23, 5048-5079. 